\title{
A single dose of nicotine proactively enhances the partial reinforcement extinction effect in the rat
}

\author{
GRIGORY GRIGORYAN and JEFFREY A. GRAY \\ The Institute of Psychiatry, London, England
}

\begin{abstract}
On the basis of previous data showing (1) the dependence of the partial reinforcement extinction effect (PREE) upon the release of noradrenaline (NA) within the hippocampal formation and (2) a long-term proactive increase in intrahippocampal NA release after systemic treatment with nicotine, it was predicted that this compound would proactively enhance the PREE. The PREE was measured in rats as running speed in the straight alley after training on continuous reinforcement (CRF) or partial reinforcement (PRF) schedules of food reward, the PREE consisting in increased resistance to extinction of the running response after PRF relative to CRF training. The prediction was confirmed, largely reflecting a nicotine-induced increase in resistance to extinction in the PRF condition, in three experiments: a single injection of nicotine $(0.8 \mathrm{mg} / \mathrm{kg}$ s.c.) preceding runway training and extinction at one trial a day, seven daily injections of nicotine preceding the same paradigm, and a single injection of nicotine preceding training and extinction at six trials a day. The observed behavioral changes occurred up to a month after nicotine administration, a time course similar to that seen in previous neurochemical experiments. The effects of the one- and seven-injection regimes on the PREE were statistically indistinguishable, also in agreement with previous neurochemical data.
\end{abstract}

If animals are repeatedly exposed to aversive events, especially in the context of reward, they develop behavioral tolerance to them (Gray, 1987). One example of this phenomenon is the partial reinforcement extinction effect (PREE; Amsel, 1962, 1992). In this paradigm, two groups of rats receive food reward for running in the straight alley, one with reward on every trial (continuous reinforcement, CRF), and the other with reward on a random $50 \%$ of the trials (partial reinforcement, PRF). The PREE consists in greater resistance to extinction (i.e., continued running for more trials when reward is discontinued) in the $P R F$ group relative to the CRF group. Much evidence suggests that the PREE reflects, at least in part, tolerance for the aversive event of frustrative nonreward (i.e., the nondelivery of expected reward) during training on the PRF schedule. Furthermore, since, for example, training on a PRF schedule gives rise to increased resistance to punishment and training on a schedule of mixed reward and punishment similarly gives rise to increased resistance to extinction (for review, see Gray, 1987), it seems that the PREE reflects a general increase in tolerance for aversive events, rather than a specific tolerance limited only to nonreward.

The PREE is reduced or abolished by electrolytic or mechanical destruction of the septal area, the hippocampal formation, and the fornix-fimbria (which carries two-way connections between the septal area and hippocampus; for references, see Feldon, Rawlins, \& Gray, 1985,

This research was supported by the Council for Tobacco Research. Correspondence should be addressed to J. A. Gray, Department of Psychology, Institute of Psychiatry, De Crespigny Park, London SE5 8AF, England. and Gray, 1982). It is also abolished by destruction of the dorsal ascending noradrenergic bundle (DNAB) by local injection of the catecholamine-specific (CA-specific) neurotoxin, 6-hydroxydopamine (6-OHDA), reducing levels of hippocampal noradrenaline (NA) by $>90 \%$ (Owen, Boarder, Gray, \& Fillenz, 1982), and by systemic administration of barbiturate or benzodiazepine anxiolytic drugs (Feldon \& Gray, 1981; Feldon, Guillamon, Gray, De Wit, \& McNaughton, 1979), an effect that has been attributed to reduced release of NA in terminals of the DNAB, especially in the hippocampal formation (Gray, 1982; Gray, McNaughton, James, \& Kelly, 1975; McNaughton et al., 1977). Against this background, therefore, we would expect that an increase in the release of NA in the hippocampus might enhance the PREE.

Such an increase in hippocampal NA release may be elicited by systemic administration of nicotine. Acutely, this compound $(0.4$ and $0.8 \mathrm{mg} / \mathrm{kg}$ s.c.) elicits an increase in hippocampal extracellular NA, measured by dialysis (Brazell, Mitchell, \& Gray, 1991), a response seen in the first 10-min sample after injection. This effect might be due to an action of nicotine at the noradrenergic cell bodies of origin of the DNAB, located in the locus coeruleus (LC), or at intrahippocampal terminals of these cells. Direct application of nicotine to the hippocampus via the dialysis probe causes an increase in extracellular hippocampal NA, again in the first 10 -min sample, but with a more rapid return to baseline than after systemic injection (Mitchell, Smith, Joseph, \& Gray, 1993). However, further experiments suggest that this is not the site at which systemic nicotine acts. In these experiments (Mitchell, 1993), systemic injections were combined with local injections of either nicotine or nicotinic 
antagonists (mecamylamine and trimethaphan) into either the LC or the hippocampus. Whereas intrahippocampal administration of the antagonists failed to alter the size of the hippocampal NA response to systemic nicotine $(0.4 \mathrm{mg} / \mathrm{kg}$ s.c. $)$, this response was blocked by administration of either mecamylamine or trimetaphan close to the LC. Thus, although intrahippocampal nicotine is capable of eliciting hippocampal NA release, at a behaviorally effective systemic dose this same response appears to be largely or entirely due to an action of nicotine at LC cell bodies.

In addition to the immediate NA-releasing effect of systemic nicotine, this treatment also produces a much longer term response.

We have observed that a single dose of nicotine $(0.8 \mathrm{mg} /$ $\mathrm{kg} \mathrm{s.c.)}$ increases the activity in the LC of the rate-limiting enzyme in CA synthesis, tyrosine hydroxylase (TH; see Smith, Joseph, \& Gray, 1991), measured 7 days later. This effect is preceded by enhanced expression of TH mRNA (Mitchell et al., 1993), measured in the LC 4-6 days after the injection. Our data strongly indicate that the longterm effects of a single nicotine dose closely approximate those of more chronic regimes (e.g., daily doses for up to 28 days; see Mitchell, Brazell, Joseph, Alavijeh, \& Gray, 1989; Mitchell et al., 1993; Smith, Joseph, \& Gray, 1991; Smith, Mitchell, \& Joseph, 1991). Thus, TH activity is increased in terminal regions of the DNAB at times proportional to the distance of the terminals from the LC (e.g., in the hippocampus, 21-28 days after injection), a pattern consistent with axonal transport of induced TH (FauconBiguet, Buda, Lamouroux, Samolyk, \& Mallet, 1986). No comparable effects were seen in dopaminergic cell bodies or terminal regions.

The effect of nicotine on TH activity in the LC is mediated by action at nicotinic receptors, since it is blocked by concomitant administration of mecamylamine, when both compounds are given either once a day for 7 days (Smith, Mitchell, \& Joseph, 1991) or once only with TH measurement 7 days later (K. Rickwood and M. H. Joseph, personal communication, February 1, 1995). In addition, there is evidence from studies of the accumulation of dihydroxyphenylalanine (DOPA), after systemic administration of a DOPA decarboxylase inhibitor, that this increase in the activity of the biochemical pathway for NA synthesis then persists in the hippocampus for at least an additional 14 days (Mitchell et al., 1989). The observed changes in TH activity appear to be limited to noradrenergic neurons whose axons travel in the DNAB (i.e., those with cell bodies in the LC), as distinct from axons in the ventral bundle, whose cell bodies are located in other brain-stem nuclei (Mitchell, Schugens, Brazell, \& Gray, 1990). Thus, a single systemic dose of nicotine acts via nicotinic receptors to increase the potential for CA synthesis selectively and enduringly in neurons of the LC. This potential was not manifest, however, as measured in the hippocampus, in basal DOPA accumulation or extracellular NA levels; rather, it appeared when a second, challenge nicotine injection was given (Mitchell et al.,
1989; Mitchell et al., 1993). Thus, this second injection increased hippocampal extracellular NA, measured by in vivo dialysis, to a greater degree in animals given a single, priming injection of nicotine 28 days previously than in controls pretreated with saline and also challenged with nicotine (Mitchell et al., 1993). In sum, a single injection of nicotine appears to increase the reactivity of LC neurons to challenge occurring as long as a month later.

If we now suppose that exposure to nonreward similarly constitutes a challenge to the LC system, as argued by Gray, Feldon, Rawlins, Owen, and McNaughton (1978; Gray, 1982), it follows from these neurochemical observations that, 3-4 weeks after a single dose of nicotine, hippocampal NA release in response to nonreward should be enhanced, and, consequently, the behavioral effects of this event should be increased. From this argument, we can predict that a single dose of nicotine should proactively affect the PREE. More specific predictions can be derived in two ways. First, it is known from behavioral analyses of the effects of frustrative nonreward (Amsel, 1992; Gray, 1987) that the greater the impact of this event, the more rapid is the course of extinction after CRF training, but, also, the greater is the increase in resistance to extinction produced by PRF training. Second, destruction of the DNAB both increases resistance to extinction after CRF training and decreases resistance to extinction after PRF training (Owen et al., 1982); therefore, enhanced function in the DNAB should produce effects opposite in sign to each of these. Both lines of argument lead to the specific predictions that, 21-42 days (Mitchell et al., 1989; Mitchell et al., 1993; Smith, Joseph, \& Gray, 1991) after a single dose of nicotine, with behavioral training interposed on either a CRF or a PRF schedule, resistance to extinction should be reduced after CRF training and further increased after PRF training, relative to placebo-treated controls.

These same predictions can be derived more indirectly from experiments on the proactive behavioral effects of experimental manipulation of the hippocampal theta rhythm, by means of electrical stimulation of the pacemaker cells for this rhythm located in the medial septal area. In a series of experiments using this technique ("theta driving"), we have shown that, applied over a series of days at a frequency of 7.7 or $8.3 \mathrm{~Hz}$, such stimulation proactively (as measured up to 40 days after the end of the period of stimulation) increases the sensitivity of the stimulated animals to frustrative nonreward; applied at a frequency of $7.5 \mathrm{~Hz}$, however, theta driving produces exactly the opposite effect (i.e., decreased sensitivity to nonreward; see Holt \& Gray, 1983; Snape, Grigoryan, Sinden, \& Gray, 1996; Williams \& Gray, 1996; Williams, Gray, Snape, \& Holt, 1989). Specifically, in the same basic alley PREE paradigm used here, Snape et al. (1995) observed that $7.7-\mathrm{Hz}$ theta driving, applied prior to any behavioral training, proactively decreased resistance to extinction after CRF training, increased resistance to extinction after PRF training, and thus enhanced the PREE. The same type of stimulation regime 
has also been reported proactively (15-30 days after stimulation) to enhance TH activity in the hippocampus (Graham-Jones, Holt, Gray, \& Fillenz, 1985). Given the data (reviewed above) implicating the DNAB projection to the hippocampus in the PREE, it is therefore possible that the proactive effects on the PREE of theta driving at these frequencies are mediated by the enhanced $\mathrm{TH}$ activity in the hippocampus (Snape et al., 1996). If so, one would expect that the induction of enhanced hippocampal TH activity by pretreatment with nicotine should also increase the PREE, giving rise to the same detailed predictions as those outlined above.

These predictions were tested in the two experiments reported here. Both experiments used food reward for running in a straight alley. They differed in that training and extinction were conducted at one trial a day (24-h intertrial interval, ITI) in Experiment 1 but at six trials a day (4- to 6-min ITI within each day) in Experiment 2. This variable was examined because previous research has shown that it can influence the effects on the PREE of certain relevant manipulations. Thus, systemic administration of the anxiolytic drugs amylobarbitone sodium (Feldon et al., 1979) and chlordiazepoxide (Feldon \& Gray, 1981) is effective in blocking the PREE at a 24-h ITI, but the PREE is blocked only in a statedependent manner at an ITI of a few minutes. In contrast, damage to the septohippocampal system blocks the PREE at both of these ITIs (Feldon \& Gray, 1979a, 1979b; Feldon et al., 1985; Henke, 1977; Rawlins, Feldon, \& Gray, 1980). In both experiments reported here, animals were given a single systemic administration of either nicotine or placebo, followed by a time table of training (on either CRF or PRF schedules) and extinction which ensured that exposure to nonreward for PRF-trained animals and exposure to extinction in both reinforcement conditions occurred at times when previous observations (Mitchell et al., 1989; Mitchell et al., 1993; Smith, Joseph, \& Gray, 1991) lead one to expect noradrenergic transmission to be enhanced in the hippocampus. Experi- ment 1 included an additional condition in which nicotine (or placebo) was administered once a day for 7 days prior to training, in order to determine whether any effect observed after the single dose of nicotine could be increased by multiple treatments with this compound. Our previous experiments indicate that the long-term neurochemical effects of 1- and 7-day nicotine pretreatment are essentially identical (Mitchell et al., 1989; Mitchell et al., 1993; Smith, Joseph, \& Gray, 1991); we therefore expected that these two regimes would similarly affect behavior to an equal extent.

\section{METHOD}

\section{Experimental Design}

Experiment 1 investigated the effects of either a single injection or seven consecutive daily injections of nicotine on the one-triala-day PREE measured over the ensuing weeks. Since there were separate placebo controls (one or seven saline injections), the study may be regarded as consisting of two parallel experiments (Experiments 1A and 1B) concerned with the proactive effects of one and seven injections of nicotine, respectively. Experiment 2 investigated the proactive effects of a single injection of nicotine only on the PREE run at six trials a day. To ensure that the key periods of acquisition and extinction coincided with observed periods of elevated hippocampal noradrenergic function caused by prior nicotine treatment (Mitchell et al., 1989; Mitchell et al., 1993; Smith, Joseph, \& Gray, 1991), the time table illustrated in Figure 1 was adopted.

\section{Animals}

Male Sprague-Dawley rats were used (Bantin and Kingman Ltd., Hull, England), weighing $250-300 \mathrm{~g}$ on arrival. They were housed 5 to a cage and maintained on a 14:10-h light:dark cycle (lights off at $2100 \mathrm{~h}$ ), with free access to water. Food was provided for $1 \mathrm{~h}$ per day after termination of behavioral testing.

\section{Apparatus}

The apparatus consisted of a straight, aluminium alley $(168 \mathrm{~cm}$ long and $17 \mathrm{~cm}$ wide, with $38 \mathrm{-cm}$ walls), in which start, run, and goal times were automatically measured to the nearest $0.01 \mathrm{sec}$ by Advance Instrument SC3 digital timers. The startbox was $22 \mathrm{~cm}$ long. The start clock was activated when the experimenter operated

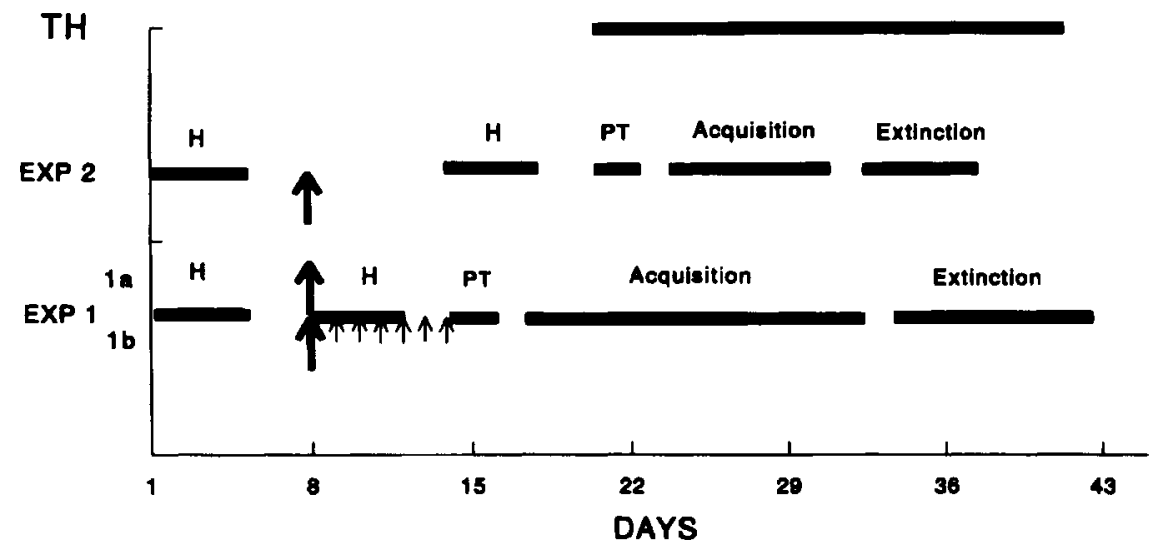

Figure 1. Timing of events in Experiments 1 (acquisition and extinction at one trial a day) and 2 (acquisition and extinction at six trials a day). Large arrow, single nicotine injection (Experiments $1 A$ and 2) or first of seven daily injections (Experiment 1B). Small arrows, remaining six injections in Experiment 1B. H, handling. PT, pretraining. TH, period of elevated hippocampal tyrosine hydroxylase activity as observed in previous experiments. 
a solenoid that released a catch allowing the startbox door to fall open. Photobeams and photocells, using visible light, were located $15 \mathrm{~cm}$ from the startbox door (terminating the start clock and activating the run clock), $97 \mathrm{~cm}$ farther along the alley (terminating the run and activating the goal clock), and $30 \mathrm{~cm}$ farther, just above the food cup in the goalbox (terminating the goal clock). Reward (45-mg Campden Instruments food pellets) was placed by hand in the $2.5-\mathrm{cm}$-diameter food cup, fixed to the floor $4 \mathrm{~cm}$ before the end of the goalbox. The subjects could be confined to the goal area by a hand-operated guillotine door.

\section{Drug Treatment}

In Experiment 1,80 rats were randomly allocated to CRF and PRF groups. They were further divided into four subgroups that received subcutaneous injections of either saline $(0.9 \% \mathrm{NaCl}$, $1.0 \mathrm{ml} / \mathrm{kg}$ ) or nicotine ([-]-nicotine hydrogen tartrate, $0.8 \mathrm{mg} / \mathrm{kg}$ [base] in $1.0 \mathrm{ml} / \mathrm{kg} 0.9 \% \mathrm{NaCl}$ ). Half of the animals from each subgroup received only single injections of saline or nicotine; the others were injected with saline or nicotine every day for 7 days. For each of the resulting eight groups (CRF-Sal 1, CRF-Nic 1, PRFSal 1, PRF-Nic 1, CRF-Sal 7, CRF-Nic 7, PRF-Sal 7, and PRFNic 7), $n=10$. Injections preceded behavioral training, as shown in Figure 1; the single-injection groups were treated on the same day as the first injection for the 7-day-injection groups.

In Experiment 2, 36 rats were randomly allocated to four subgroups (each with $n=9$ ), which were given either CRF or PRF training orthogonally combined with saline or nicotine treatment. Only single injections were made (drug parameters as in Experiment 1).

\section{Training Procedures}

Pretraining consisted of handling the rats in groups of 4 for $10 \mathrm{~min} /$ day for 10 days followed by 3 days of exposure to the alley. On the first day, the rats were placed in the alley in groups of 4 for $10 \mathrm{~min}$, with the doors open and 50 reward pellets scattered throughout the alley. On the second day, they were placed in the alley in pairs for two 5 -min periods, with 25 pellets scattered throughout the alley each time. On the third day, they received two 2-min placements singly; each time 10 reward pellets were scattered throughout the alley. Acquisition began immediately after pretraining.

There were 16 days of acquisition in Experiment 1 (one trial a day) and 8 days of acquisition in Experiment 2 (six trials a day), followed by 10 days of extinction in Experiment 1 and 6 days in Experiment 2. In Experiment 2, the rats were run in squads of 4 with a 4- to 6-min ITI. Reward was 20 pellets in Experiment 1 and 10 pellets in Experiment 2. For CRF, reward was given on every trial; for PRF, reward was given on $50 \%$ of trials (half of each day's trials in Experiment 2). The PRF schedule was quasi-random-with the limitation that not more than two rewarded (R) or nonrewarded (N) trials occurred consecutively - and balanced so that, as far as possible, transitions between $\mathrm{R}$ and $\mathrm{N}(\mathrm{R}-\mathrm{R}, \mathrm{R}-\mathrm{N}, \mathrm{N}-\mathrm{R}$, and $\mathrm{N}-\mathrm{N})$ occurred with equal probability within (Experiment 2) and across days. The schedule used in Experiment 1 (one trial a day) was as follows: R, N, R, N, R, N, R, N, R, N, N, R, R, N, N, R. Extinction trials were identical to acquisition trials, except that no animal was ever rewarded. The extinction criterion was three successive trials on which a subject failed to reach the goal within $100 \mathrm{sec}$. On nonrewarded trials during both acquisition and extinction, goalbox confinement time was $30 \mathrm{sec}$.

\section{Statistics}

The data (start, run, goal, and total speeds, $1 / \mathrm{sec}$ ) were analyzed separately for acquisition and extinction by split-plot analyses of variance (ANOVAs) using GENSTAT (Rothamsted, England). The between-subjects factors were schedule (CRF vs. PRF), drug (nicotine vs. saline) and, for Experiment 1 only, duration ( 1 day vs.
7 days of drug treatment). Days and trials (for Experiment 2) were within-subjects factors. Linear and quadratic trends across days were extracted by orthogonal polynomials and compared by $t$ test, on the basis of the appropriate error terms in the ANOVAs.

\section{RESULTS}

The major predictions under test concern interactions between drug (pretreatment with nicotine or saline) and schedule (CRF or PRF), especially during extinction. The presentation of results will accordingly concentrate upon such interactions; other effects will be reported only insofar as they affect interpretation of the phenomena of central interest.

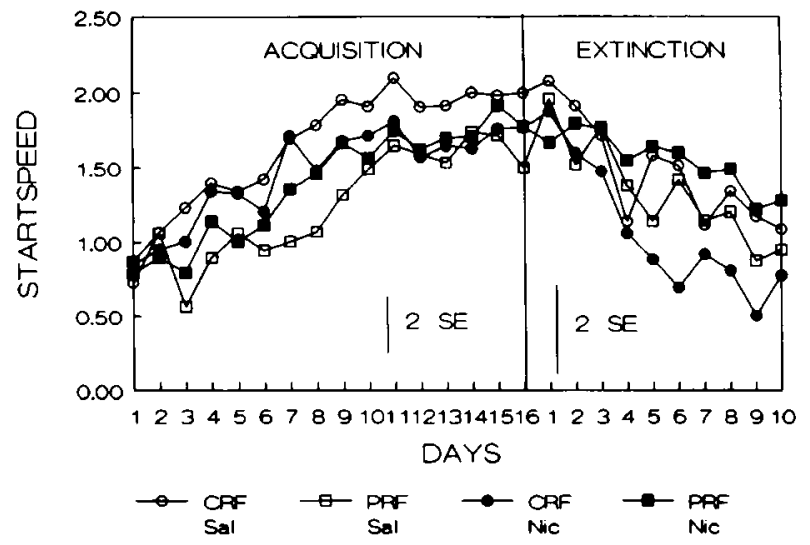

Figure 2. Start speeds (1/sec) during acquisition and extinction at one trial a day in Experiment 1, using data combined across Experiments $1 \mathrm{~A}$ (single nicotine injection) and $1 \mathrm{~B}$ (seven daily injections). CRF, continuous reinforcement. PRF, partial reinforcement. Sal, saline controls. Nic, nicotine treatment. 2 SE, 2 standard errors based on the days $x$ drug $X$ schedule interactions in the analyses of variance of acquisition and extinction data.

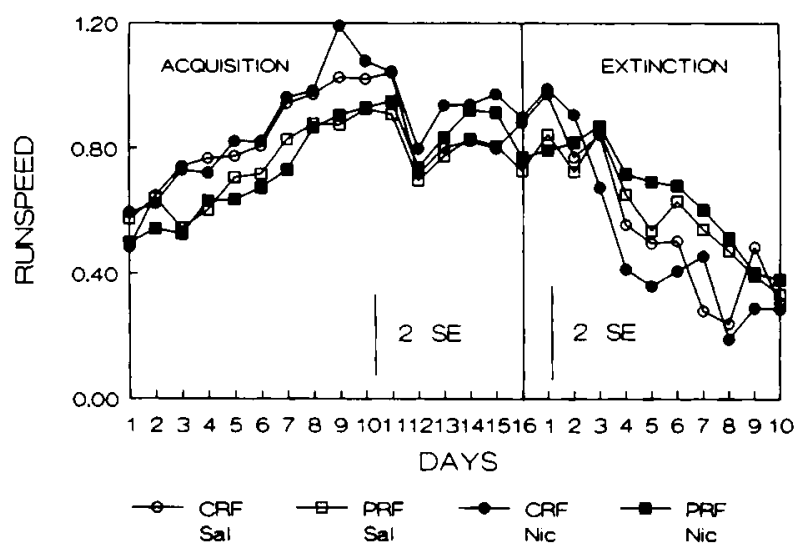

Figure 3. Run speeds in Experiments 1A and 1B combined. CRF, continuous reinforcement. PRF, partial reinforcement. Sal, saline controls. Nic, nicotine treatment. $2 S E, 2$ standard errors based on the days $x$ drug $x$ schedule interactions in the analyses of variance of acquisition and extinction data. 


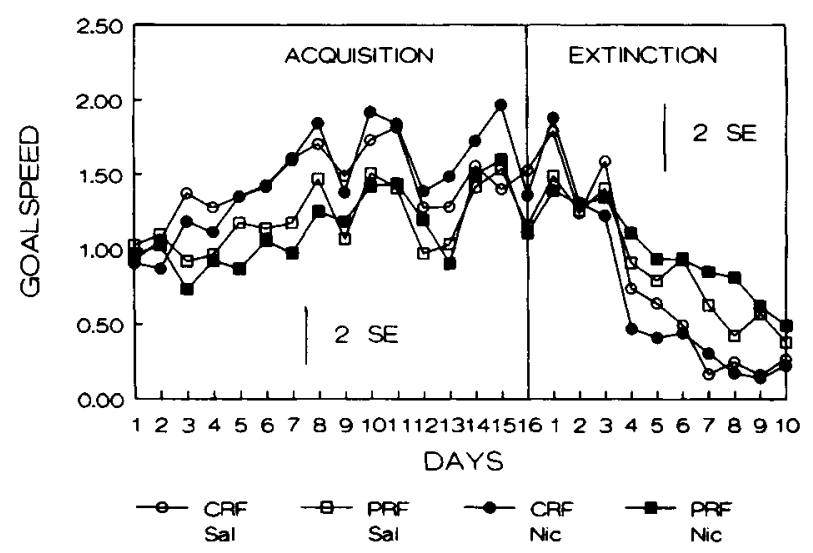

Figure 4. Goal speeds in Experiments $1 \mathrm{~A}$ and $1 \mathrm{~B}$ combined. CRF, continuous reinforcement. PRF, partial reinforcement. Sal, saline controls. Nic, nicotine treatment. 2 SE, 2 standard errors based on the days $X$ drug $X$ schedule interaction in the analyses of variance of acquisition and extinction data.

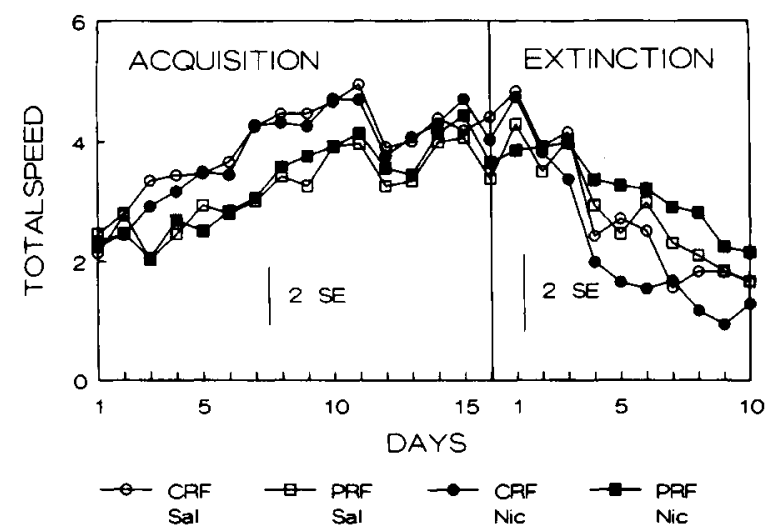

Figure 5. Total speeds in Experiments $1 \mathrm{~A}$ and $1 \mathrm{~B}$ combined. CRF, continuous reinforcement. PRF, partial reinforcement. Sal, saline controls. Nic, nicotine treatment. $2 S E, 2$ standard errors based on the days $X$ drug $X$ schedule interactions in the analyses of variance of acquisition and extinction data.

\section{Experiments 1A and 1B (One Trial a Day) Analyzed Together}

There were no significant interactions involving duration with both drug and schedule, in either the acquisition or the extinction data. Thus, any influence of pretreatment with nicotine on the difference between CRF- and PRF-trained animals was independent of the duration of such pretreatment ( 1 or 7 days). The data are therefore presented collapsed across this variable in Figures 2-5, for all alley sections (start, run, and goal), as well as total speeds.

Acquisition. All groups displayed increasing speeds over the course of acquisition in all three alley sections. This pattern was more pronounced with CRF training than with PRF training, as supported statistically, for example, by the significant effects of schedule $[F(1,72)=$
$6.89, p<.05]$ and days $\times$ schedule $[F(15,1080)=3.41$, $p<.001]$, in the total speeds. These effects of schedule did not interact with drug, except in the start section, where there was a significant linear component in the days $\times$ drug $\times$ schedule interaction $[F(1,1080)=5.80$, $p<.02]$. This interaction reflects abolition by nicotine of the difference in gain of speed between the saline-treated CRF and PRF groups (the former gaining speed more rapidly; Figure 2). Importantly, there were no significant group differences at the end of acquisition that might complicate interpretation of the extinction data.

Extinction. There was clear evidence of a PREE in all alley sections (Figures 2-5), manifest in both the linear and quadratic components of the days $X$ schedule interaction (Table 1). Graphically, the PREE was substantially more pronounced in the nicotine-treated animals than in the controls. This was confirmed statistically in the total speeds, for which both the linear and the quadratic components of the days $\times$ drug $\times$ schedule interaction were significantly modified by nicotine pretreatment [linear, $F(1,648)=3.52$; quadratic, $F(1,648)=$ 4.18 ; both $p s<.05]$. Comparison of the relevant linear coefficients by $t$ test showed that there was a significant difference between CRF and PRF groups (i.e., a PREE) in the nicotine condition $[t(72)=4.51, p<.001]$, but not in the saline condition, confirming the graphical display (Figure 5). Similar comparisons of the quadratic coefficients provided evidence of a small PREE within the saline-treated groups $[t(72)=2.22, p<.05]$ and also displayed a significant difference between the nicotineand saline-treated PRF groups $[t(72)=4.35, p<.001]$. Scrutiny of the data from the separate alley sections

Table 1

Statistical Evidence for the PREE in Experiments $1 \mathrm{~A}$ and $1 \mathrm{~B}$ and in Experiment 1A + Experiment $1 B$ Combined:

Results of $F$ Tests on the Schedule $\times$ Days Interactions for Start, Run, Goal, and Total Speeds During Extinction and for the Linear and Quadratic Components of These Interactions

\begin{tabular}{llccc}
\hline & & \multicolumn{3}{c}{ Experiment } \\
\cline { 3 - 5 } Speed & \multicolumn{1}{c}{ Effect } & 1A & 1B & $1 \mathrm{~A}+1 \mathrm{~B}$ \\
\hline Start & Schedule $\times$ Days & n.s. & n.s. & n.s. \\
& Linear & n.s. & $12.72 \ddagger$ & $5.40^{*}$ \\
& Quadratic & $12.72 \ddagger$ & n.s. & $7.42 \dagger$ \\
Run & Schedule $\times$ Days & $3.20 \ddagger$ & $1.98^{*}$ & $3.74 \ddagger$ \\
& Linear & n.s. & $11.28 \ddagger$ & $6.91 \ddagger$ \\
& Quadratic & $23.78 \ddagger$ & n.s. & $21.54 \ddagger$ \\
Goal & Schedule $\times$ Days & $2.03^{*}$ & $4.23 \ddagger$ & $5.33 \ddagger$ \\
& Linear & n.s. & $23.40 \ddagger$ & $22.98 \ddagger$ \\
& Quadratic & $10.18 \ddagger$ & $10.91 \ddagger$ & $21.08 \ddagger$ \\
Total & Schedule $\times$ Days & $3.86 \ddagger$ & $3.11 \ddagger$ & $5.38 \ddagger$ \\
& Linear & n.s. & $19.73 \ddagger$ & $19.65 \ddagger$ \\
& Quadratic & $27.41 \ddagger$ & $5.10^{*}$ & $26.32 \ddagger$ \\
\hline
\end{tabular}

Note-For Experiments $1 \mathrm{~A}$ and $1 \mathrm{~B}$ data, $d f \mathrm{~s}=9,324$ for the schedule $X$ days interaction, and $d f \mathrm{~s}=1,324$ for the linear and quadratic components of these interactions. For Experiment $1 \mathrm{~A}+$ Experiment $1 \mathrm{~B}$ data combined $d f \mathrm{~s}=9,648$ for the schedule $\times$ days interactions, and $d f \mathrm{~s}=1,648$ for the linear and quadratic components of these interactions. n.s. $=$ nonsignificant. ${ }^{*} p<.05$. $\dagger p<.01$. $\ddagger p<.001$. 
showed that this effect of nicotine was largely due to changes in start speeds (Figure 2). For these, there was a significant linear component of the days $\times$ drug $\times$ schedule interaction $[F(1,648)=6.97, p<.01]$. The $t$ tests revealed a pattern similar to the one in total speeds: a significant PREE within the nicotine-treated groups $[t(72)=$ $3.55, p<.001]$ but not the saline groups, and a significant difference between the nicotine- and saline-treated PRF groups $[t(72)=2.14, p<.05]$. The graphically similar effects in the run and goal sections, however, failed to attain statistical significance.

\section{Experiments 1A and 1B (One Trial a Day) Analyzed Separately}

From the analysis of the combined data, it is clear that nicotine pretreatment (irrespective of its duration) proactively enhanced the PREE, as measured by total speeds, and especially in the start section. The four-way interactions between days, drug, schedule, and duration in the combined analysis fell well short of significance for all alley sections, the highest $[F(9,648)=1.63]$ being for goal speeds. Nonetheless, we also analyzed the data separately for the 1- and 7-day nicotine conditions, so as to ascertain whether these treatments had each on its own enhanced the PREE.
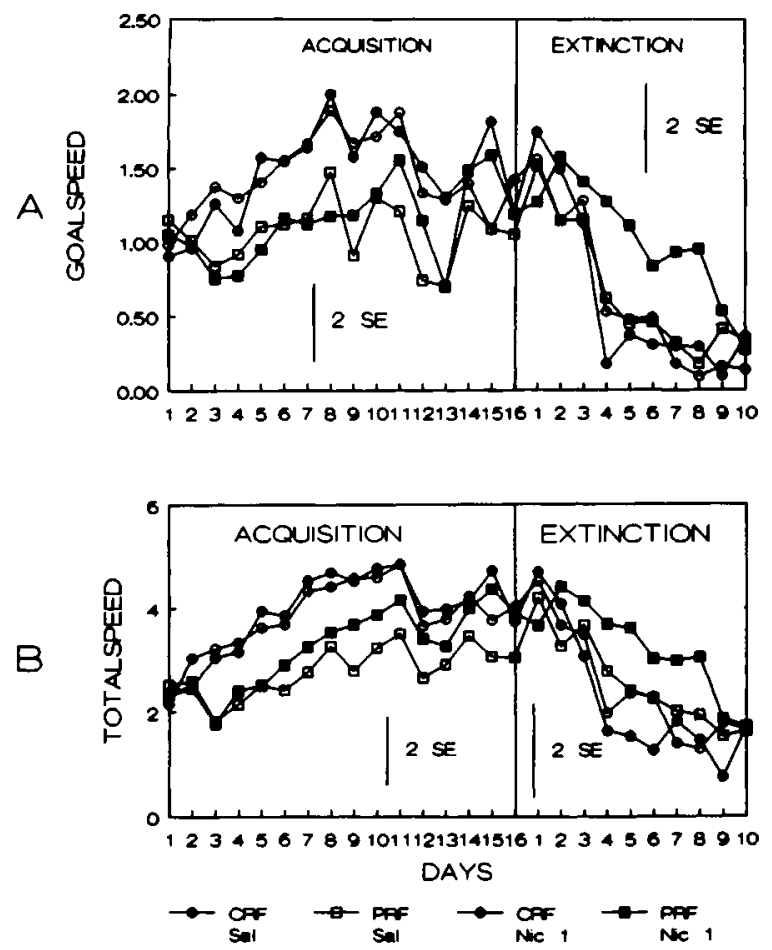

Figure 6. Goal (A) and total (B) speeds during acquisition and extinction at one trial a day in Experiment $1 \mathrm{~A}$ (single nicotine injection). CRF, continuous reinforcement. PRF, partial reinforcement. Sal, saline controls. Nic, nicotine treatment. $2 S E, 2$ standard errors based on the days $x$ drug $\times$ schedule interactions in the analyses of variance of acquisition and extinction data.
Table 2

Significant Results of $\boldsymbol{F}$ Tests on Quadratic Components of the Schedule $\times$ Drug $\times$ Days Interaction in the Extinction Data and Significant Results of $t$ Tests on the Subsequent Comparisons of the Quadratic Coefficients in Experiment 1A

\begin{tabular}{|c|c|c|c|}
\hline Section & $F(1,324)$ & Comparison & $t(36)$ \\
\hline \multirow[t]{2}{*}{ Goal } & $13.77 \dagger$ & PRF-N versus PRF-S & $3.05^{*}$ \\
\hline & & PRF-N versus $C R F-N$ & $4.89 \dagger$ \\
\hline \multirow[t]{2}{*}{ Total } & $8.77^{*}$ & PRF-N versus PRF-S & $2.75^{*}$ \\
\hline & & PRF-N versus CRF-N & 5.80 \\
\hline
\end{tabular}

Note-PRF $=$ partial reinforcement. $\mathrm{CRF}=$ continuous reinforcement. $\mathrm{N}=$ nicotine. $\mathrm{S}=$ saline. $.{ }^{*} p<.01 . \quad \dagger p<.001$.

Single injection of nicotine. The acquisition data revealed no significant interactions involving both drug and schedule, and there were no significant asymptotic differences between groups that might complicate interpretation of the extinction data. There was evidence of an overall PREE, especially in the quadratic components of the days $\times$ schedule interactions (Table 1 ). Nicotine pretreatment modified the PREE in the goal section (Figure 6A), as shown by the significant quadratic component in the interaction between drug, schedule, and days $[F(1,324)=$ $13.77, p<.001]$, and in total speeds (Figure 6B), for which there was again a significant quadratic component in this interaction $[F(1,324)=8.77, p<.003]$. As is clear from Figure 6 , the PREE in both goal and total speeds was due to differences between the CRF and PRF groups within the nicotine condition only. Comparisons of the relevant quadratic coefficients for both goal and total speeds (Table 2) confirmed this impression and showed that the nicotine-produced enhancement of the PREE was largely due to increased resistance to extinction in the PRF condition. In the start and run sections, drug did not interact with schedule, although a graphically similar pattern (data not shown) was apparent.

Injections of nicotine for 7 days. In this data set, there were again no significant interactions during acquisition involving both schedule and drug, and there were no significant asymptotic acquisition differences affecting interpretation of the extinction data. Analysis of the latter showed evidence of an overall PREE in all alley sections, which was more apparent in the linear components of the days $\times$ schedule interactions than (as was the case after a single injection of nicotine) in the quadratic components (Table 1). Nicotine pretreatment modified the PREE in the start speeds, for which the linear component of the days $\times$ drug $\times$ schedule interaction was significant $[F(1,324)=4.42, p<.05]$. As shown in Figure 7A, and as confirmed by $t$ tests comparing the relevant linear coefficients (Table 3 ), the difference between the CRF and PRF groups (i.e., the PREE) was significant in the nicotine condition but not in the saline condition; this effect was largely due to a nicotine-induced increase in resistance to extinction in the PRF condition. A similar pattern was marginally significant in the total speeds [linear component of the days $\times$ drug $\times$ schedule 

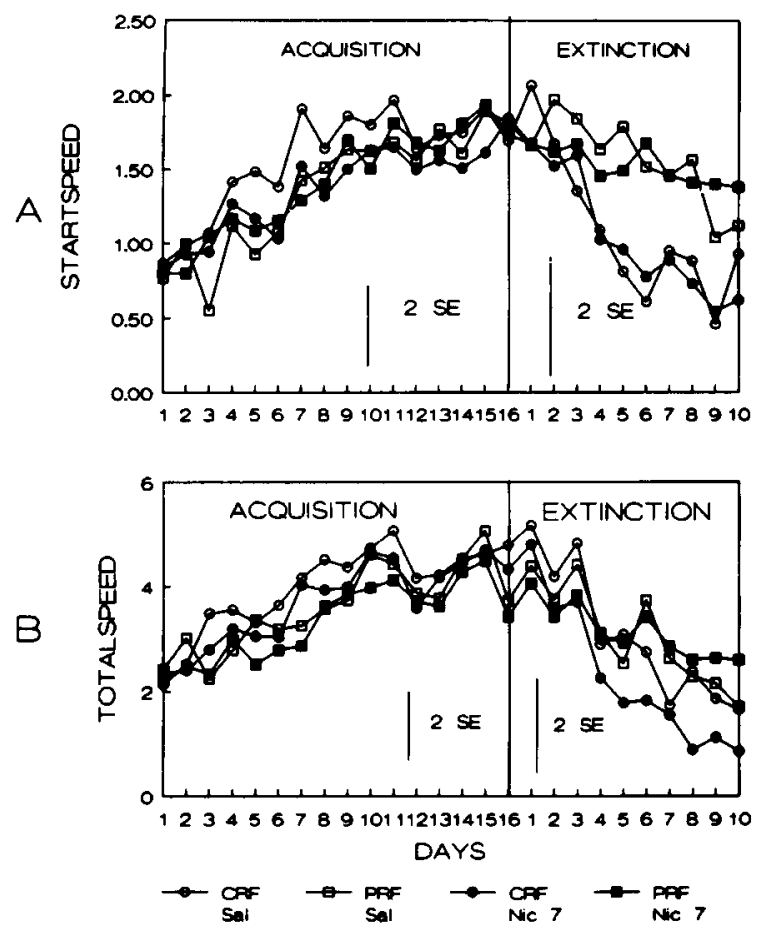

Figure 7. Start (A) and total (B) speeds during acquisition and extinction at one trial a day in Experiment 1B (seven nicotine injections). CRF, continuous reinforcement. PRF, partial reinforcement. Sal, saline controls. Nic, nicotine treatment. $2 S E, 2$ standard errors based on the days $x$ drug $X$ schedule interactions in the analyses of variance of acquisition and extinction data.

interaction, $F(1,324)=3.60, p=.06$; see Figure 7B and Table 3]. There was no evidence of such a pattern, either graphically (data not shown) or statistically in the run or goal sections.

\section{Experiment 2 (Six Trials a Day)}

The results for all alley sections and for total speeds are presented in Figures 8-11.

Acquisition. Schedule had significant effects, which persisted to the end of training. In the start section (Figure 8), CRF groups gained speed faster than did PRF groups and were faster at the end of acquisition [schedule, $F(1,32)=4.36, p<.05$; days $\times$ schedule, $F(7,224)=$ $2.16, p<.05]$. A similar pattern in the run section (Figure 9 ) was supported by the days $\times$ schedule interaction $[F(7,224)=3.11, p<.005]$ and the very pronounced pattern of the same kind in the goal section (Figure 10) by effects of schedule $[F(1,32)=15.17, p<.001]$ and days $\times$ schedule $[F(7,224)=10.17, p<.001]$. Nicotine pretreatment retarded the gain of speed over days of acquisition, as reflected in the linear components of the days $\times$ drug interactions in start, run, and total speeds $[F \mathrm{~s}(1,224)=3.59,7.78$, and $4.81, p \mathrm{~s}<.06, .01$, and .05 , respectively]. However, there were no significant interactions between schedule and drug, thus permitting reasonably straightforward interpretation of the extinction data.
Extinction. Overall, a PREE was evident in the run and goal sections, where speeds for PRF-trained animals were faster across all days of extinction $[F \mathrm{~s}(1,32)=6.39$ and 6.28, respectively, $p \mathrm{~s}<.02]$. There was evidence of modification of the PREE by pretreatment with nicotine in all alley sections, as well as in total speeds. These effects emerged as significant (or, in the goal section, marginally significant) linear components in the days $\times$ drug $\times$ schedule interactions (Table 4). Comparisons of the relevant linear coefficients by $t$ test (Table 4) confirmed the graphical impression that these significant interactions reflected a nicotine-induced enhancement of the PREE (i.e., the PRF group losing speed more slowly than the CRF group) due to an increase in resistance to extinction in the PRF condition.

In the run section, analysis of the trial-by-trial data (Figure 12) revealed further evidence of a nicotineinduced modification of the PREE, in the shape of a significant interaction between trials, drug, and schedule $[F(5,960)=2.62, p<.025]$. This effect reflected the fact that the decrease in run speeds over the six daily extinction trials (averaged across all days of extinction) was slower in the nicotine-treated PRF group than in the corresponding saline group.

\section{DISCUSSION}

The major results of the experiments can be summarized as follows. In Experiment 1, the magnitude of the PREE, tested at one trial a day, was proactively increased by pretreatment with nicotine; the nicotine effect was essentially equivalent whether pretreatment took the form of a single injection or seven daily injections (except that the former regime principally affected goal speeds and the latter affected start speeds); and the effect was largely due to increased resistance to extinction in nicotine-pretreated animals trained on the PRF schedule. In Experiment 2, run at six trials a day (ITI $=4-6 \mathrm{~min}$ ), the magnitude of the PREE was again increased by pretreatment with nicotine (a single injection), and, again, the nicotine effect was largely due to increased resistance to extinction in the PRF condition. Interpretation of these effects was, in no case, complicated by drug-induced changes in asymptotic acquisition performance.

Table 3

Significant Results of $\boldsymbol{F}$ Tests on Linear Components of the Schedule $\times$ Drug $\times$ Days Interactions in the Extinction Data and Significant Results of $t$ Tests on the Subsequent Comparisons of the Linear Coefficients in Experiment $1 B$

\begin{tabular}{llll}
\hline Section & $F(1,324)$ & \multicolumn{1}{c}{ Comparison } & $t(36)$ \\
\hline Start & $4.42 \dagger$ & PRF-N versus PRF-S & $2.35 \dagger$ \\
& & PRF-N versus CRF-N & $3.04 \ddagger$ \\
Total & $3.60^{*}$ & PRF-N versus CRF-N & $4.05 \S$ \\
& & PRF-N versus PRF-S & $2.2 \dagger$ \\
\hline
\end{tabular}

Note-PRF $=$ partial reinforcement. $\mathrm{CRF}=$ continuous reinforcement. $\mathrm{N}=$ nicotine. $\mathrm{S}=$ saline. ${ }^{*} p=.06 . \quad \dagger p<.05 . \quad \ddagger p<.01$. $\S p<.001$. 


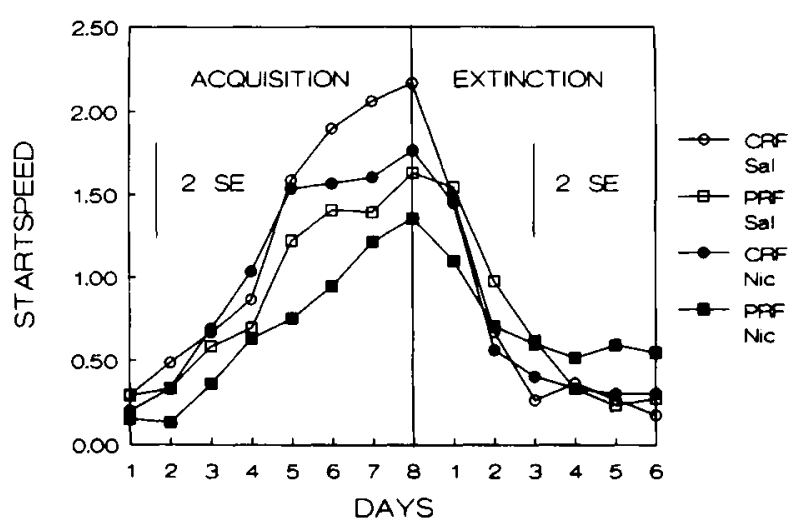

Figure 8. Start speeds during acquisition and extinction in Experiment 2 (six trials a day). CRF, continuous reinforcement. PRF, partial reinforcement. Sal, saline controls. Nic, nicotine treatment. $2 S E$, 2 standard errors based on the days $X$ drug $X$ schedule interactions in the analyses of variance of acquisition and extinction data.

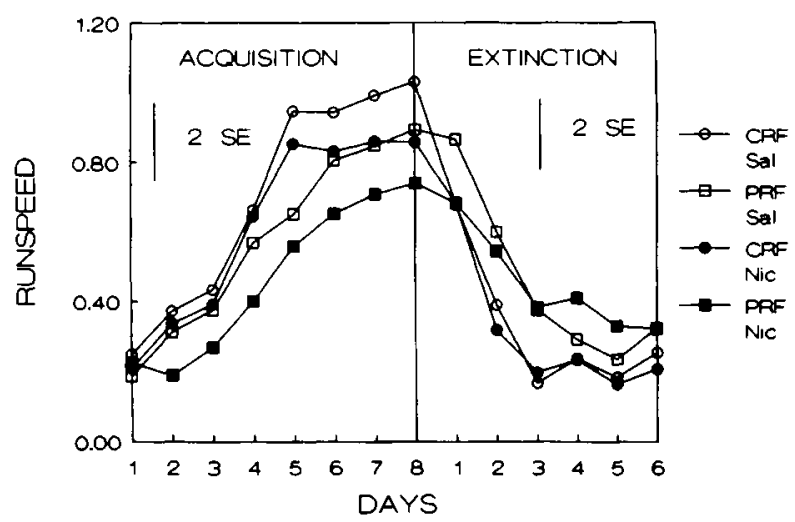

Figure 9. Run speeds during acquisition and extinction in Experiment 2 (six trials a day). CRF, continuous reinforcement. PRF, partial reinforcement. Sal, saline controls. Nic, nicotine treatment. $2 S E$, 2 standard errors based on the days $\times$ drug $\times$ schedule interactions in the analyses of variance of acquisition and extinction data.

The main prediction that these experiments set out to test - namely, that a single injection of nicotine would proactively increase the PREE, with behavioral training and testing taking place many days later (Figure 1)-was well buttressed by previous data and theoretical argument (see Introduction). In the absence of this theoretical background, the a priori probability of the prediction would certainly have been regarded as very low. Thus, confirmation of the prediction in Experiments $1 \mathrm{~A}$ and 2 lends considerable weight to the theoretical arguments from which it was derived.

Experiment 1B provides further confirmation of the predictions tested. Like Experiment 1 A, this measured the PREE at one trial a day. It differed from Experiment $1 \mathrm{~A}$ only in the number of nicotine injections: seven versus one. Since our previous observations (Mitchell et al., 1989; Mitchell et al., 1993; Smith, Joseph, \& Gray,
1991) show that the proactive effects of seven daily injections of nicotine upon parameters of noradrenergic functioning do not differ from those of one such injection, we expected a similar equality of effect upon the PREE. The joint analysis of the data from the oneinjection and 7-day-injection regimes confirmed this expectation, the two data sets being statistically indistinguishable. To the extent that the separate analyses of the results of Experiments $1 \mathrm{~A}$ and $1 \mathrm{~B}$ revealed differences between the two injection regimes, these suggested that, if anything, the effects of the single injection were slightly more substantial than those of seven injections. Thus, as in other experiments from our laboratory (Abdulla et al., in press; Mitchell et al., 1993), the observed changes after nicotine, even when injected repeatedly over 7 days, were not due principally to a chronic dosing regime but rather reflect delayed effects of a single administration of this compound.

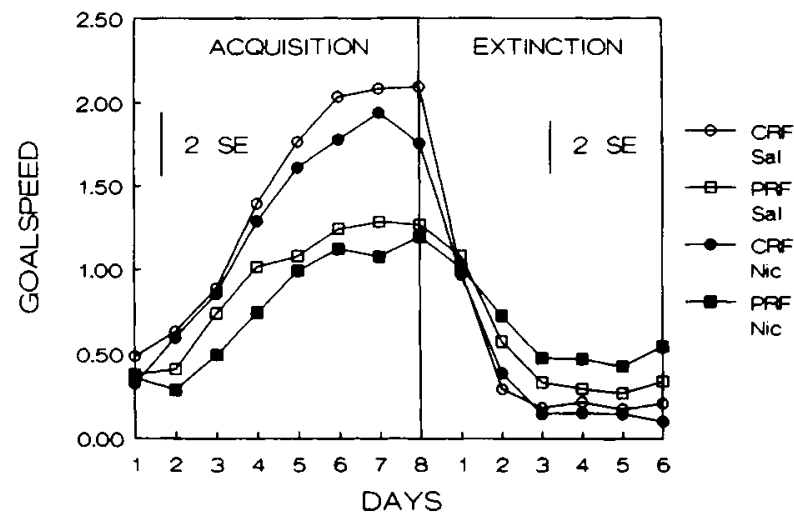

Figure 10. Goal speeds during acquisition and extinction in Experiment 2 (six trials a day). CRF, continuous reinforcement. PRF, partial reinforcement. Sal, saline controls. Nic, nicotine treatment. $2 S E, 2$ standard errors based on the days $\times$ drug $\times$ schedule interactions in the analyses of variance of acquisition and extinction data.

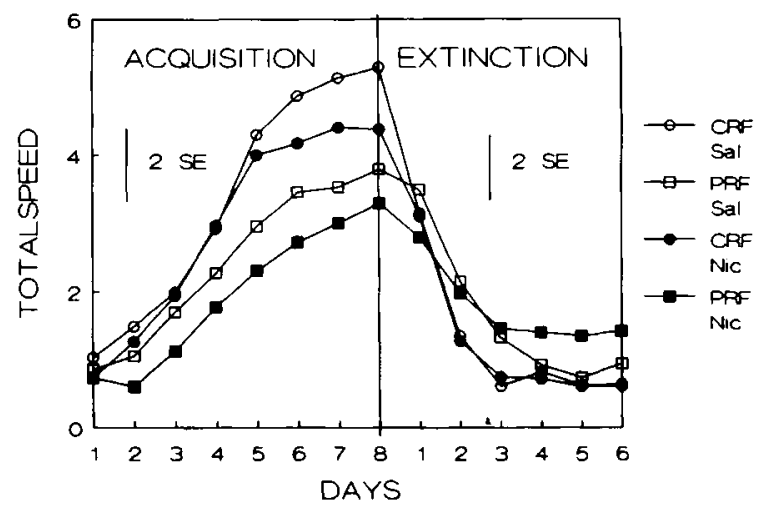

Figure 11. Total speeds during acquisition and extinction in Experiment 2 (six trials a day). CRF, continuous reinforcement. PRF, partial reinforcement. Sal, saline controls. Nic, nicotine treatment. $2 S E, 2$ standard errors based on the days $\times$ drug $\times$ schedule interactions in the analyses of variance of acquisition and extinction data. 
Table 4

Significant Results of $F$ Tests on Linear Components of the Schedule $\times$ Drug $\times$ Days Interactions in the Extinction Data and Significant Results of $t$ Tests on the Subsequent Comparisons of the Linear Coefficients in Experiment 2

\begin{tabular}{llll}
\hline Section & $F(1,160)$ & \multicolumn{1}{c}{ Comparison } & $t(32)$ \\
\hline Start & $5.57 \dagger$ & PRF-N versus PRF-S & $4.05 \S$ \\
& & PRF-N versus CRF-N & $2.04 \dagger$ \\
Run & $4.52 \dagger$ & PRF-N versus PRF-S & $2.73 \ddagger$ \\
& & PRF-S versus CRF-S & $2.26 \dagger$ \\
Goal & $3.66^{*}$ & PRF-N versus CRF-N & $2.16 \dagger$ \\
Total & $7.49 \ddagger$ & PRF-N versus PRF-S & $2.68 \dagger$ \\
& & PRF-N versus CRF-N & $2.54 \dagger$ \\
\hline
\end{tabular}

Note-PRF $=$ partial reinforcement. $\mathrm{CRF}=$ continuous reinforcement. $\mathrm{N}=$ nicotine. $\mathrm{S}=$ saline. $\quad{ }^{*} p=.06 . \quad \dagger p<.05 . \quad \ddagger p<.01$. $\S p<.001$

The major difference between the effects of the singleinjection and 7-day injection regimes in the one-trial-aday paradigm lies in the detailed pattern by which both proactively enhanced the PREE. The single injection did this by influencing principally the quadratic component of the shape of the extinction curve, whereas the multipleinjection regime affected principally the linear component. It is difficult to evaluate the psychological significance of this difference. In addition, the single injection had stronger effects upon extinction in the goal section of the alley, whereas the multiple-injection regime had stronger effects in the start section. A possible interpretation of this pattern of results is that single nicotine administration affects responses to stronger degrees of frustration (thought to be greater in the goal section than the start section of a straight alley; Amsel, 1992) than does the multiple-injection regime. An effect of this kind might arise if the additional six injections (whether of saline or nicotine) that differentiate the multiple-injection regime from the single-injection regime led to adaptational changes, which then influenced the degree of frustration experienced during acquisition of the running response. Transfer of adaptational effects between different stressors in this way has been reported in other paradigms (e.g., Brown \& Wagner, 1964; Chen \& Amsel, 1982). Interestingly, the combined analysis of the data from the two sets of experiments reflected these separate effects of each drug regime on its own, the influence of nicotine pretreatment being apparent in both the start section and (though not clearly) the goal section and in both the linear and the quadratic components of the shape of the extinction curve.

The proactive enhancement of the PREE by nicotine is evidently robust, having been observed in all three experiments reported here, involving both a one-trial-a-day paradigm (Experiments $1 \mathrm{~A}$ and $\mathrm{BB}$ ) and a multiple-trial paradigm (Experiment 2). In contrast, the effects of anxiolytic drugs, given during behavioral training and/or testing, are clearly apparent only in the one-trial-a-day paradigm (Feldon \& Gray, 1981; Feldon et al., 1979). Thus, the proactive effects of nicotine upon the PREE appear to be wider than those of the anxiolytics, resembling in this respect those of damage to the septohippocampal system (Feldon \& Gray, 1979a, 1979b; Feldon et al., 1985; Henke, 1977; Rawlins et al., 1980). There is, however, a point of difference between the effects on the PREE of nicotine observed here and those of either damage to (references above) or stimulation of (Snape et al., 1996) the septohippocampal system. The latter are mediated about equally by changes in resistance to extinction in both the CRF and the PRF conditions (taking opposite directions in the two cases). The effects observed here, in contrast, were largely (though not entirely) mediated by changes in resistance to extinction in the PRF condition. It is as yet difficult to evaluate the importance of this difference.

A link between the proactive action of nicotine and the functioning of the septohippocampal system is strongly suggested by the chain of argument (see Introduction) from which the effects observed here were predicted. Since damage to the septohippocampal system has the opposite effects (weakening the PREE) to those observed here, this link presumably takes the form that nicotine proactively enhances the activity of the septohippocampal system. As outlined in the Introduction, an effect of this kind could be mediated by a nicotine-induced proactive increase in intrahippocampal noradrenaline release, as observed by Mitchell et al. (1993). This hypothesis is consistent with the fact that damage to the DNAB, like damage to the septohippocampal system, impairs the PREE (Owen et al., 1982). If the hypothesis is correct, it should be possible to detect a proactive enhancement of hippocampal neural transmission some weeks after nicotine administration. We have recently observed just such an effect (Hamid, Dawe, Gray, \& Stephenson, in press). In these experiments, we measured, in anesthetized rats, the effects of a challenge dose of nicotine $(0.4 \mathrm{mg} / \mathrm{kg})$ on the slope of the excitatory postsynaptic potential (EPSP), recorded in the dentate gyrus in response to perforant path

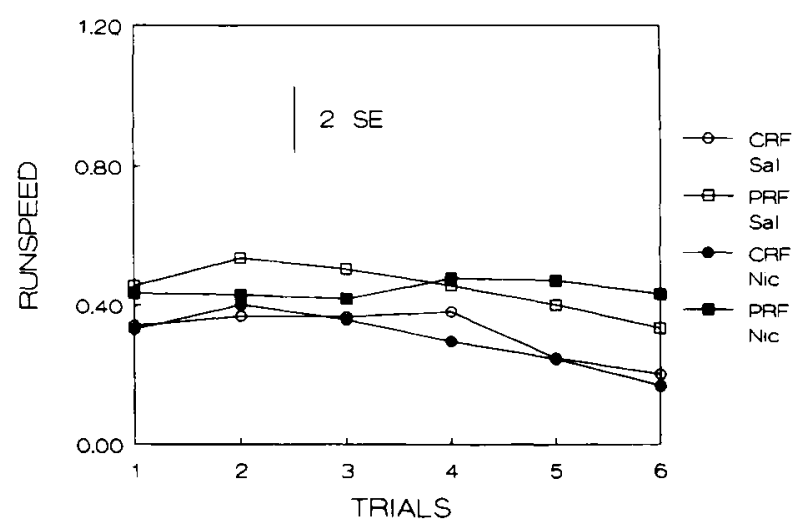

Figure 12. Run speeds in Experiment 2 (six trials a day) as a function of trials averaged across days of extinction. $2 S E, 2$ standard errors based on the trials $X$ drug $\times$ schedule interaction in the analysis of variance. CRF, continuous reinforcement. PRF, partial reinforcement. Sal, saline controls. Nic, nicotine treatment. 
stimulation. The slope of the EPSP was enhanced by the challenge dose of nicotine, and this effect was blocked by concomitant administration ( $30 \mathrm{~min}$ before nicotine) of the beta-adrenoceptor antagonist, propranolol, consistent with previous reports that (1) systemic nicotine causes hippocampal release of noradrenaline (Mitchell et al., 1993) and (2) bath application of noradrenaline to hippocampal slices potentiates the response of dentate granule cells to perforant path stimulation (Stanton, Mody, \& Heinemann, 1989). In addition, our data indicate that this effect of acute nicotine on the EPSP slope occurred only in animals pretreated with systemic nicotine $(0.8 \mathrm{mg} / \mathrm{kg}$ daily for 7 days, as in the present Experiment 1B) given 3 weeks previously, not in controls pretreated with saline. It seems possible, therefore, that the final common pathway for the proactive effects upon the PREE of nicotine, as well as those of theta-driving septal stimulation at frequencies of $7.7-8.3 \mathrm{~Hz}$ (GrahamJones et al., 1985; Snape et al., 1996), includes an increase in the transmission of information within the hippocampal system consequent upon increased intrahippocampal release of noradrenaline (Mitchell et al., 1993; Segal, 1977).

\section{REFERENCES}

Abdulla, F., Bradbury, E., Calaminici, M.-R., Lippiello, P. M., Wonnacott, S., Gray, J. A., \& Sinden, J. D. (in press). Relationship between up-regulation of nicotine binding sites in rat brain and delayed cognitive enhancement observed after chronic or acute nicotinic receptor stimulation. Psychopharmacology.

AMSEL, A. (1962). Frustrative nonreward in partial reinforcement and discrimination learning: Some recent history and a theoretical extension. Psychological Review, 69, 306-328.

Amsel, A. (1992). Frustration theory. Cambridge: Cambridge University Press.

Brazell, M. P., Mitchell, S. N., \& Gray, J. A. (1991). Effect of acute administration of nicotine on in vivo release of noradrenaline in the hippocampus of freely moving rats: A dose-response and antagonist study. Neuropharmacology, 30, 823-833.

Brown, R. T., \& WAGNER, A. R. (1964). Resistance to punishment and extinction following training with shock or nonreinforcement. Journal of Experimental Psychology, 68, 503-507.

CHEN, J. S., \& AMSEL, A. (1982). Habituation to shock and learned persistence in pre-weaning, juvenile and adult rats. Journal of Experimental Psychology: Animal Behavior Processes, 8, 113-130.

Faucon-Biguet, N., Buda, M., Lamouroux, A., SAmolyK, D., \& MALLET, J. (1986). Time course for the changes in tyrosine hydroxylase mRNA in rat brain and adrenal medulla after a single injection of reserpine. European Molecular Biology Organisation Journal, 5, 287-291.

Feldon, J., \& Gray, J. A. (1979a). Effects of medial and lateral septal lesions on the partial reinforcement extinction effect at one trial a day. Quarterly Journal of Experimental Psychology, 31, 653-674.

FELDON, J., \& GRAY, J. A. (1979b). Effects of medial and lateral septal lesions on the partial reinforcement extinction effect at short intertrial intervals. Quarterly Journal of Experimental Psychology, 31, 675-690.

FELDON, J., \& GRAY, J. A. (1981). The partial reinforcement extinction effect after treatment with chlordiazepoxide. Psychopharmacology, 73, 269-275.

Feldon, J., Guillamon, A., Gray, J. A., De Wit, H., \& McNaughton, N. (1979). Sodium amylobarbitone and responses to nonreward. Quarterly Journal of Experimental Psychology, 31, 19-50.

Feldon, J., RaWlins, J. N. P., \& GRAY, J. A. (1985). Fornix-fimbria section and the partial reinforcement extinction effect. Experimental Brain Research, 58, 435-439.

Graham-Jones, S., Holt, L., Gray, J. A., \& Fillenz, M. (1985). Lowfrequency septal stimulation increases tyrosine hydroxylase activity in the hippocampus. Pharmacology, Biochemistry \& Behavior, 23, 489-493.

GraY, J. A. (1982). The neuropsychology of anxiety: An enquiry into the function of the septo-hippocampal system. Oxford: Oxford University Press.

GraY, J. A. (1987). The psychology of fear and stress. Cambridge: Cambridge University Press.

Gray, J. A., Feldon, J, RaWlins, J. N. P., Owen, S., \& McNaughton, N. (1978). The role of the septo-hippocampal system and its noradrenergic afferents in behavioural responses to nonreward. In K. Elliott \& J. Whelan (Eds.), Functions of the septo-hippocampal system (pp. 275-300). Amsterdam: Elsevier.

Gray, J. A., McNaughton, N., James, D. T. D., \& Kelly, P. H. (1975). Effect of minor tranquillizers on hippocampal theta rhythm mimicked by depletion of forebrain noradrenaline. Nature, 258, 424-425.

Hamid, S., Dawe, G., Gray, J. A., \& Stephenson, J. D. (in press). Nicotine-induced long-lasting potentiation in the rat dentate gyrus (Abstract). British Journal of Pharmacology.

HENKE, P. G. (1977). Dissociation of the frustration effect and the partial reinforcement extinction effect after limbic lesions in rats. Journal of Comparative \& Physiological Psychology, 91, 1032-1038.

HoLT, L., \& GRAY, J. A. (1983). Septal driving of the hippocampal theta rhythm produces a long-term, proactive and non-associative increase in resistance to extinction. Quarterly Journal of Experimental Psychology, 35B, 97-118.

MCNaughton, N., James, D. T. D., Stewart, J., Gray, J. A., Valero, I., \& DrewnowsKI, A. (1977). Septal driving of hippocampal theta rhythm as a function of frequency in the male rat: Effects of drugs. Neuroscience, 2, 1019-1027.

Mrtchell, S. N. (1993). Role of the locus coeruleus in the noradrenergic response to a systemic administration of nicotine. Neuropharmacology, 32, 937-949.

Mitchell, S. N., Brazell, M. P., Joseph, M. J., Alavijeh, M. S., \& GraY, J. A. (1989). Regionally specific effects of acute and chronic nicotine on rates of catecholamine and indoleamine synthesis in rat brain. European Journal of Pharmacology, 167, 311-322.

Mitchell, S. N., Schugens, M. M., Brazell, M. P., \& Gray, J. A. (1990). Nicotine-induced catecholamine synthesis after lesions to the dorsal or ventral noradrenergic bundle. European Journal of Pharmacology, 179, 383-391.

Mitchell, S. N., Smith, K. M., Joseph, M. H., \& Gray, J. A. (1993). Increases in tyrosine hydroxylase messenger RNA in the locus coeruleus after a single dose of nicotine are followed by timedependent increases in enzyme activity and noradrenaline release Neuroscience, 56, 989-997.

Owen, S., Boarder, M., Gray, J. A., \& Fillenz, M. (1982). Acquisition and extinction of continuously and partially reinforced running in rats with lesions of the dorsal noradrenergic bundle. Behavioural Brain Research, 5, 11-41.

Rawlins, J. N. P., Feldon, J., \& Gray, J. A. (1980). The effects of hippocampectomy and of fimbria section upon the partial reinforcement extinction effect in rats. Experimental Brain Research, 38, 273-283.

SEGAL, M. (1977). The effects of brainstem priming stimulation on interhemispheric hippocampal responses in the awake rat. Experimental Brain Research, 29, 553-565.

Smith, K. M., Joseph, M. H., \& Gray, J. A. (1991). A single dose of nicotine is sufficient to increase tyrosine hydroxylase in noradrenergic neurones. In A. Adlkofer \& K. Thurau (Eds.), Effects of nicotine on biological systems: Advances in pharmacological sciences (pp. 345-350). Basel: Birkhauser Verlag.

SMith, K. M., Mitchell, M. H., \& JosePh, M. H. (1991). Effects of chronic and subchronic nicotine on tyrosine hydroxylase activity in noradrenergic and dopaminergic neurones in the rat brain. Journal of Neurochemistry, 57, 1750-1756.

Snape, M., Grigoryan, G., Sinden, J. D., \& Gray, J. A. (1996). Dependence of the proactive behavioral effects of theta-driving septal 
stimulation on stimulation frequency and behavioral experience: 2. Continuously and partially reinforced running. Psychobiology, 24, 22-32.

Stanton, P. K., Mody, I., \& Heinemann, U. (1989). A role for $N$-methyl$D$-aspartate receptors in norepinephrine-induced long-lasting potentiation in the dentate gyrus. Experimental Brain Research, 77, 517-530. Williams, J. H., \& GRAY, J. A. (1996). Dependence of the proactive behavioral effects of theta-driving septal stimulation on stimulation frequency and behavioral experience: 1 . Leverpress experiments. Psychobiology, 24, 9-21.
Williams, J. H., Gray, J. A., Snape, M., \& Holt, L. (1989). Long-term effects of septohippocampal stimulation on behavioural responses to anxiogenic stimuli. In P. Tyrer (Ed.), Psychopharmacology of anxiety (British Association for Psychopharmacology Monograph No. 11, pp. 80-108). Oxford: Oxford University Press.

(Manuscript received July 31, 1995; revision accepted for publication October 26,1995 .)

\section{Erratum}

Snape, M., Grigoryan, G., Sinden, J. D., \& Gray, J. A. Dependence of the proactive behavioral effects of theta-driving septal stimulation on stimulation frequency and behavioral experience: 2 . Continuously and partially reinforced running. Psychobiology, 1996, 24(1), 22-32.On page 31, the reference to Owen, Boarder, Gray, and Fillenz (1982) should read as follows:

Owen, S., Boarder, M., Gray, J. A., \& Fillenz, M. (1982). Acquisition and extinction of continuously and partially reinforced running in rats with lesions of the dorsal noradrenergic bundle. Behavioural Brain Research, 5, 11-41. 